\title{
Uptake of home-based syphilis and HIV testing among male partners of pregnant women in western Kenya
}

\author{
Jennifer Mark, MPH ${ }^{1}$, John Kinuthia, MBChB, MMed, MPH ${ }^{2,3,4}$, Alison C. Roxby, MD, MSc ${ }^{7}$, \\ Daisy Krakowiak, MPH, PhD ${ }^{1}$, Alfred Osoti, MBChB, MMed, MPH ${ }^{1,3,4}$, Barbra A. Richardson, \\ $\mathrm{PhD}^{5}$, Molly Ann Gone, BSc${ }^{4}$, Victor Asila, BSc ${ }^{4}$, Saloni Parikh, BS ${ }^{6}$, and Carey Farquhar, \\ MD, MPH ${ }^{1,7,8}$ \\ ${ }^{1}$ Department of Epidemiology, University of Washington; Seattle, Washington, USA \\ ${ }^{2}$ Departments of Research and Programs, Kenyatta National Hospital; Nairobi, Kenya \\ ${ }^{3}$ Department of Reproductive Health, Kenyatta National Hospital; Nairobi, Kenya \\ ${ }^{4}$ Department of Obstetrics and Gynaecology, University of Nairobi; Nairobi, Kenya \\ ${ }^{5}$ Department of Biostatistics, University of Washington; Seattle, Washington, USA \\ ${ }^{6}$ Department of Computer Science and Engineering, University of Washington; Seattle, \\ Washington, USA \\ ${ }^{7}$ Department of Medicine, University of Washington; Seattle, Washington, USA \\ ${ }^{8}$ Department of Global Health, University of Washington; Seattle, Washington, USA
}

\begin{abstract}
Background-Few men are tested for syphilis or HIV during their partner's pregnancy, a highrisk period for HIV and syphilis transmission. Offering home-based rapid testing of syphilis to couples during pregnancy can support prevention efforts to reduce transmission of sexually transmitted diseases and adverse pregnancy outcomes.
\end{abstract}

Methods-We assessed men's uptake of paired (separate tests, single blood draw) point-of-care syphilis and HIV tests within a randomized controlled trial of pregnant women who received clinic or home partner HIV testing. We evaluated acceptance of paired HIV-syphilis testing during pregnancy or at 6 months postpartum, and evaluated whether addition of syphilis testing affected the uptake of HIV testing among men.

Results-Out of 601 women, we were unable to meet 101 male partners, and 180 tested before syphilis tests were available. Paired syphilis and HIV testing was offered at home to 80 men during pregnancy and to 230 men postpartum. For syphilis, $93 \%$ of men agreed to test during pregnancy and $98 \%$ agreed postpartum. For paired syphilis and HIV testing, $91 \%$ of men tested for both during pregnancy and $96 \%$ tested postpartum. Before syphilis test introduction, $96 \%$ of men

Corresponding Author: Name: Jennifer Mark, jmark55@uw.edu, Tel: +1-206-619-8830, Fax: Please contact for fax information, Address: Department of Epidemiology, 1959 NE Pacific Street, Seattle, WA 98195.

Conflicts of Interest: None

Presentations: Presented in part at the 8th International AIDS Society, 2015, Vancouver, Canada, Abstract \#TUPPD0105. 
accepted HIV testing, compared to $95 \%$ of men who accepted HIV testing when paired testing was offered.

Conclusions-Uptake of syphilis and HIV testing was high among male partners offered couple testing at home. Introducing syphilis testing did not adversely affect HIV testing among men. Point-of-care diagnostics outside facilities can increase testing of male partners who rarely accompany women to antenatal clinics.

\section{Keywords}

syphilis; HIV; rapid test; male partner; pregnant women

\section{INTRODUCTION}

Engaging male partners of pregnant women is an important, but often neglected, pillar of maternal and child health program success in sub-Saharan Africa. Despite recent efforts to engage men during their partners' pregnancy, rates of male clinic-based antenatal attendance, including couple HIV testing ${ }^{1,2}$ and antenatal syphilis treatment, ${ }^{3-5}$ are generally low. The effects of male engagement during female pregnancy have mainly been studied in the context of prevention of mother-to-child-transmission of HIV (PMTCT), in which male partner involvement within antenatal care increases male HIV testing ${ }^{6,7}$ and identifies more HIV-discordant couples. ${ }^{8,9}$ Furthermore, male engagement improves women's uptake of PMTCT services ${ }^{1,10}$ and reduces infant HIV acquisition and mortality. ${ }^{11}$ Offering male partner syphilis education and testing could lead to similar program improvements and reduce the 930,000 maternal infections and 350,000 syphilis-associated perinatal deaths that occur globally each year. ${ }^{12,13}$

The advent of rapid and inexpensive point-of-care (POC) tests for both HIV and syphilis has expanded test coverage, especially in areas with difficult-to-reach populations or without access to laboratory diagnostics. ${ }^{14}$ These novel tools make elimination of congenital syphilis possible, but will likely require high screening coverage and effective partner management strategies. ${ }^{15}$ In Kenya, presumptive treatment of male partners of women who tested positive for syphilis during pregnancy led to a reduction of stillbirths and low birth weight babies compared to children of untreated male partners. ${ }^{16}$ Syphilis testing can also benefit existing HIV PMTCT efforts as demonstrated in Uganda and Zambia, where addition of syphilis tests led to small but significant increases in HIV testing as well as increased uptake of maternal single-dose nevirapine prophylaxis and combination antiretroviral treatment. ${ }^{17}$ Syphilis and HIV testing during pregnancy are highly cost-effective interventions during pregnancy ${ }^{18,19}$ and can be scaled up in low- and high-resource settings. ${ }^{20}$

In contrast to low rates of antenatal clinic-based HIV (12-15\%) $1,10,21$ and syphilis testing of male partners $(<2 \%),{ }^{3}$ home-based STI education and testing has been shown to be highly acceptable ( $>83 \%$ HIV test uptake) and feasible in several settings in Africa. ${ }^{8,22,23}$

Additionally, cost-effectiveness models in sub-Saharan Africa suggest that paired HIV and syphilis testing strategies, including single-cartridge duplexed HIV-syphilis tests, are cost saving and result in fewer disability-adjusted life-years lost compared to HIV-only testing programs. ${ }^{19}$ We examined whether paired POC syphilis and HIV testing at home were 
acceptable to male partners of pregnant women as part of a randomized controlled trial. We determined uptake of syphilis and HIV tests during pregnancy and at 6 months postpartum and conducted a comparison of HIV test uptake among male partners before and after the addition of syphilis testing.

\section{METHODS}

This was an observational cohort study; study participants were male partners of women who participated in a randomized controlled trial (RCT) of home-based partner education and testing (HOPE) that enrolled 601 pregnant women presenting to a first antenatal clinic visit at Kisumu County Hospital in Nyanza Province, Kenya from September 2013 to June $2014 .{ }^{8}$ Pregnant women were eligible to enroll in the study if they were married or cohabiting, had not experienced intimate partner violence within the preceding month, aged $\geq 14$ years and living within 40 kilometers of the hospital. Consenting women were randomized to receive either: (1) home-based partner education and HIV testing with their male partner (HOPE intervention, $n=306$ ); or (2) a male partner invitation letter for clinicbased HIV testing at the maternal and child health clinic (control, $n=295$ ). Couples received counseling in the home about family health, including HIV, STI symptoms and clinic-based treatment, syphilis POC tests, exclusive breast feeding, facility delivery and family planning, and were encouraged, but not required, to test together as a couple with mutual disclosure. HIV testing included standard pretest and posttest counseling.

In the larger randomized controlled trial, staff encountered male partners of the intervention arm at the home-based HOPE intervention visit during pregnancy. Outcomes for all participants of the RCT were then assessed at a home-based 6-month postpartum follow-up visit, at which time men of the control arm were first encountered. This exit visit included HIV testing to determine final infection status. ${ }^{8}$ As this is an area of high HIV incidence, men were classified as having unknown HIV status if they did not self-identify as HIV positive.

We assessed uptake of a POC treponemal syphilis test (SD Bioline Syphilis 3.0; Standard Diagnostics Inc., South Korea) when conducted concurrently with rapid HIV testing. First, we examined uptake of first-time offer of syphilis testing within the intervention arm (during pregnancy) and control arm (6 months postpartum) of the RCT. Due to test unavailability at the study start, syphilis tests were offered to intervention participants after some men received HIV-only testing and education during pregnancy. Second, we assessed whether the addition of syphilis testing affected HIV testing, by comparing HIV test uptake during pregnancy before and after syphilis testing was added to the home-based HOPE HIV couple education and testing intervention.

\section{Home-based couple education and paired HIV-syphilis testing visit}

After syphilis testing became available, men could accept HIV and syphilis POC testing together (paired), HIV testing only, syphilis testing only, or no testing. If both tests were chosen, syphilis and HIV testing and results were conducted at the same time utilizing a single finger-prick blood draw. Study staff referred men who tested positive for syphilis to maternal and child health clinics for cost-free intramuscular penicillin treatment per World 
Health Organization guidelines. All HIV-infected participants were referred to HIV comprehensive care clinics per Kenyan national protocol.

At the 6-month postpartum visit, all participants were offered an exit interview and paired HIV and syphilis testing. Therefore, men in the control arm received STI education and a first offer of syphilis testing at this time, while men in the intervention arm were offered repeat testing for HIV and syphilis.

\section{Data collection and analysis}

Study staff surveyed couples via password-protected mobile phone-based questionnaires in Open Data Kit (ODK) software. ${ }^{24}$ Among men enrolled in the study, first-time syphilis test uptake data proportions were calculated, and HIV test uptake before and after syphilis test introduction was analyzed using chi-square tests. Data were analyzed using Stata 12 (StataCorp LLC, College Station, TX).

\section{Human Subjects}

The study protocol was approved by Kenyatta National Hospital Ethics Review Committee in Nairobi and the Institutional Review Board at the University of Washington. Written informed consent was obtained in Swahili, Luo, or English for all participants prior to enrollment.

\section{RESULTS}

\section{Study Population}

Among 601 pregnant women enrolled in the parent randomized controlled trial, 101 male partners (17\%) were not located or did not agree to discuss participation: 46 men (15\%) in the HOPE arm and 55 men (19\%) in the control arm were never able to be contacted, and 32 women and partners in the control arm (11\%) were lost before the postpartum testing visit. Of the remaining 500 male partners, 180 men were enrolled and tested for only HIV before the syphilis test was available. After syphilis testing was available, 320 male partners were enrolled in the study. (Figure 1). Of these men, 80 in the HOPE arm were offered paired syphilis and HIV testing at home during their partners' pregnancy, and 240 in the control arm were offered paired syphilis-HIV testing at home during a postpartum visit. Test uptake data were missing for 10 men in the control arm.

The median age of participating men was 30 [interquartile range (IQR) 27 to 35] (Table 1). All were married and 95\% were living together with their female partners. Most men had children prior to the study-enrolled pregnancy, as only 20 (25\%) and $36(22 \%)$ were firsttime parents in the intervention and control arms, respectively. Among experienced parents with at least one previous child, $21 \%$ (11 of 52) of men in the intervention arm and $16 \%$ (20 of 127) of men in the control arm attended any ANC visit during the last pregnancy that occurred prior to female study enrollment.

HIV POC testing was familiar and acceptable to this population of men, and self-disclosure of female HIV testing to their male partners was reported by men to be high. A total of $93 \%$ (74 of 80) of men in the intervention arm and 88\% (143 of 172) of men in the control arm 
reported previous experience with HIV testing prior to the home-based couple visit. A total of $8 \%$ (6 of 80) of men in the intervention arm and 11\% (18 of 163) of men in the control arm reported known HIV-positive status. Most men reported knowing their female partner's HIV status during pregnancy $(81 \%, 65$ of 80$)$ and postpartum $(93 \%, 149$ of 230). Of these men, $17 \%$ (11 of 65 ) in the intervention arm and 14\% (22 of 158) in the control arm had known HIV-positive female partners.

\section{Male partner uptake of syphilis \& HIV testing and the effect of introducing syphilis testing}

Among male partners who received a first-time offer of paired syphilis and HIV testing during pregnancy or at 6-months postpartum, $93 \%$ (74 of 80 ) of men accepted syphilis testing in the intervention arm and $98 \%$ (226 of 230) of men in the control arm (Table 2). Reasons for refusing syphilis testing were not available for the three HIV-positive men who refused syphilis testing during the intervention in pregnancy, but were captured among all study participants completing 6-month postpartum visits (intervention and control arms). Out of 10 men (1 HOPE, 9 control) who refused syphilis testing, 3 men indicated that they "Did not think it is necessary" and 7 men "Did not want to know." Refusal options that were not cited included, "Not understanding the test," "Fearing a test finger prick," or "Female partner unwilling to couple test or share results".

Syphilis test positivity among male partners was low in this population with $1.4 \%$ (1 of 74) of intervention arm men and $0.9 \%$ ( 2 of 226) of men in the control arm testing positive (Table 2). All three syphilis-positive men were in syphilis-discordant couples as their partners tested negative for syphilis. The man who tested positive for syphilis during pregnancy in the intervention arm had also declined HIV testing and did not know his female partner's HIV status. At 6 months postpartum, he reported that he had sought clinicbased STI services for syphilis treatment during pregnancy and accepted couple HIV testing at the 6-month postpartum visit. He was HIV-negative and his female partner was HIVpositive. The two male partners in the control arm who tested positive for syphilis postpartum were both HIV-negative and their female partners were negative for both HIV and syphilis. Among female partners of all male participants, 1.3\% (4 of 310) of couples offered paired testing during pregnancy or postpartum had tested positive for syphilis (1 intervention, 3 control). Overall, syphilis testing revealed a total of 2 intervention and 5 control couples affected by syphilis; all 7 couples were syphilis-discordant.

Considering paired syphilis and HIV testing, first-time uptake of paired syphilis and HIV testing was high among men of unknown HIV status who were eligible for paired testing (Table 2). In the intervention arm, out of 74 male partners of unknown HIV status, 91\% (67) accepted both HIV and syphilis testing, 5.4\% (4) accepted syphilis testing only, 4.1\% (3) accepted HIV testing only and none refused both tests. In the control arm, out of 211 men with unknown HIV status, 96\% (204) accepted both HIV and syphilis testing, 1.4\% (3) accepted syphilis testing only, none accepted HIV testing only and $1.9 \%$ (4) refused both tests. We also assessed the acceptability of syphilis testing among HIV-positive men who were ineligible for HIV testing. Among 25 men who reported known HIV-positive status, 3 of $6(50 \%)$ of men in the HOPE arm and all $19(100 \%)$ of men in the control arm accepted syphilis testing. 
Lastly, we examined the effect of adding POC syphilis testing to the home-based HIV test (Table 3). Among male partners who were eligible for HIV testing, 96\% (151 of 158) accepted HIV testing prior to syphilis test introduction compared to $95 \%$ (70 of 74) of men who accepted HIV testing when both HIV and syphilis testing were offered (Chi-square, $\mathrm{p}=0.6$ ).

\section{DISCUSSION}

This study demonstrated high acceptability of both syphilis and HIV testing among male partners of pregnant women when offered together at home in a couple testing environment. Home-based testing visits were accepted by more than $80 \%$ of enrolled couples in both pregnancy and postpartum. Of those reached by the intervention, uptake of syphilis testing was nearly as high or higher (93\% and 98\%) compared to HIV test uptake (95\% and 96\%) among men with unknown HIV status during early pregnancy and at 6 months postpartum, respectively. Consequently, most men with unknown HIV status accepted both syphilis and HIV testing (91\% during pregnancy and $96 \%$ postpartum) when offered together at home. We had small numbers of HIV positive men, who were more likely to decline a syphilis test, which we speculate was due to desire to avoid a blood draw since HIV status was known.

The acceptability of syphilis and HIV testing found in this study is higher than findings in prior studies. In neighboring Uganda, where syphilis prevalence is higher (6.2\% among males and $4.0 \%$ among females), $81 \%$ of male partners who sought clinic-based testing following invitation letters accepted both HIV and syphilis testing, $17 \%$ accepted only HIV testing, and $1.7 \%$ accepted only syphilis testing. ${ }^{3}$ In rural Brazil, home-based POC syphilis and HIV testing of were highly acceptable and feasible, with $85 \%$ accepting paired HIVsyphilis testing. ${ }^{25}$ The higher uptake in our study may be a result of participants' experience with previous HIV testing, as well as the positive effects of home-based couple testing on male partner HIV test uptake. Home-based couple HIV testing is desired by male partners ${ }^{23}$ and has resulted in two-fold greater ( $87 \%$ vs. $39 \%$; $R R=2.10)$ HIV test uptake among men during pregnancy compared to clinic-based invitation of the control group. ${ }^{8}$ Our qualitative research also shows that home-based testing enables close rapport between counselors and partners in a comfortable home environment that can be more conducive to POC testing than clinic-based options (Krakowiak, submitted).

In addition to our finding that syphilis testing was acceptable to men of unknown HIV status, it was also acceptable to most HIV-positive men. Among the 25 previously diagnosed HIV-positive male partners, testing acceptance was 50\% (3 of 6) among men in the intervention arm and $100 \%$ (19 of 19) among men in the control arm. Given the higher prevalence of syphilis among HIV-positive men (6.4\%) and women $(3.2 \%)$ in Kenya, ${ }^{26}$ prioritizing syphilis testing among HIV-positive individuals and their partners before and during pregnancy could be a cost-effective approach to preventing antenatal syphilis transmission and adverse pregnancy outcomes and support overall PMTCT program success. ${ }^{27}$ Although the prevalence of syphilis in this study was low, it is notable that all 3 men and 4 women testing positive for syphilis were in a syphilis-discordant couple, indicating a high yield for preventing maternal-to-child transmission of syphilis with successful treatment and repeat testing of both partners. Identification and treatment of male 
partners throughout pregnancy is critical to successful joint elimination of mother-to-child transmission of syphilis and HIV. This is particularly important among discordant couples with a syphilis positive male partner in order to prevent adverse pregnancy outcomes or neonatal infections, which occur with $52 \%$ greater frequency among untreated mothers with syphilis infection compared to women without syphilis infection. ${ }^{28}$

We were unable to determine whether men were more motivated to test for syphilis during pregnancy (to benefit the unborn child) versus in the postpartum period (for their own benefit), as uptake of the test was similar and high during both periods. This suggested that home-based couple counseling, rather than timing, mattered. Men received counseling messages that emphasized both their own general health and the health of their partner and infant as part of counseling encouraging them to test for syphilis and HIV.

Since the syphilis POC test became available halfway through the study, our study design allowed assessment of the effect of introducing syphilis testing to HIV testing. HIV test acceptability among men of unknown HIV status did not change and remained high at 95\%, indicating that syphilis test introduction did not negatively affect male partner HIV testing. Similarly, a systematic review found no adverse effects on existing female rapid HIV-testing rates among 6 studies that introduced maternal rapid syphilis testing. ${ }^{29}$ In some study sites (Peru, Cambodia, Uganda, and Zambia), maternal HIV testing increased after paired HIVsyphilis testing was introduced.

A strength of this research is the home-based couple testing approach to increasing male partner engagement, which directly recruited male partners of pregnant women. We previously demonstrated that offering home-based couple HIV testing increases HIV testing and identifies more male-positive HIV discordant couples during pregnancy compared to clinic-based couple HIV testing. ${ }^{8,22,23}$ This current study shows that home-based couple syphilis testing is equally acceptable, and this family-centered approach identifies syphilis discordant couples during a high-risk time for adverse pregnancy outcomes. Additional approaches involving male partners, such as combined phone-tracing and home visits in a Malawi study, have been shown to increase clinic-based male participation from 50\% to $72 \%$ compared to invitation letters alone. ${ }^{7}$ A combination of these approaches may provide a cost-effective strategy for scaling up couple-based antenatal services.

Given the success of this strategy and its modest cost, scale-up should be considered in countries seeking to improve HIV and syphilis testing in the antenatal period. Further implementation research would be useful to characterize the minimum factors that may be key to the successful recruitment of male partners, such as financial incentives and use of male counselors.

In summary, home-based POC syphilis testing was as acceptable as HIV testing among male partners of pregnant women in a setting where previous HIV test experience was high and male antenatal attendance was low. New POC diagnostics allow increased access to testing outside of antenatal clinics and may assist men to connect with clinic-based STI treatment. Pairing syphilis and HIV testing is an acceptable and potentially cost-effective approach to traditional syphilis control strategies. Diagnosing and successfully treating syphilis among 
male partners of pregnant women can improve male partner health and reduce adverse pregnancy outcomes, including neonatal syphilis and stillbirths.

\section{Acknowledgments}

Sources of grant funding: This study was funded by National Institutes of Health [R01 HD 075108], NIH Fogarty International Center Northern Pacific Global Health Fellows Program [Research Training Grant \#R25-TW009345], Boren International Fellowships, and International and Foreign Language Education [\#P015B100200].

We thank the participants and Kisumu County Hospital for their support; without it this research would not be possible. We also would like to thank our partners at the Kenya National AIDS and STI Control Program.

\section{References}

1. Msuya SE, Mbizvo EM, Hussain A, Uriyo J, Sam NE, Stray-Pedersen B. Low male partner participation in antenatal HIV counselling and testing in northern Tanzania: implications for preventive programs. AIDS Care. 2008; 20(6):700-709. [PubMed: 18576172]

2. Mohlala BK, Boily MC, Gregson S. The forgotten half of the equation: randomized controlled trial of a male invitation to attend couple voluntary counselling and testing. AIDS. 2011; 25(12):15351541. [PubMed: 21610487]

3. Kizito D, Woodburn PW, Kesande B, et al. Uptake of HIV and syphilis testing of pregnant women and their male partners in a programme for prevention of mother-to-child HIV transmission in Uganda. Trop Med Int Health. 2008; 13(5):680-682. [PubMed: 18331533]

4. Osman NB, Challis K, Cotiro M, Nordahl G, Bergstrom S. Maternal and fetal characteristics in an obstetric cohort in Mozambique. Afr J Reprod Health. 2000; 4(1):110-119. [PubMed: 11000714]

5. Hira SK, Bhat GJ, Chikamata DM, et al. Syphilis intervention in pregnancy: Zambian demonstration project. Genitourin Med. 1990; 66(3):159-164. [PubMed: 2370060]

6. John-Stewart GC, Mbori-Ngacha D, Payne BL, et al. HV-1-specific cytotoxic T lymphocytes and breast milk HIV-1 transmission. J Infect Dis. 2009; 199(6):889-898. [PubMed: 19434932]

7. Rosenberg NE, Mtande TK, Saidi F, et al. Recruiting male partners for couple HIV testing and counselling in Malawi's option B+ programme: an unblinded randomised controlled trial. Lancet HIV. 2015; 2(11):e483-491. [PubMed: 26520928]

8. Krakowiak D, Kinuthia J, Osoti AO, et al. Home-Based HIV Testing Among Pregnant Couples Increases Partner Testing and Identification of Serodiscordant Partnerships. J Acquir Immune Defic Syndr. 2016; 72(Suppl 2):S167-173. [PubMed: 27355505]

9. Osoti AO, John-Stewart G, Kiarie J, et al. Home visits during pregnancy enhance male partner HIV counselling and testing in Kenya: a randomized clinical trial. AIDS. 2014; 28(1):95-103. [PubMed: 23942059]

10. Farquhar C, Kiarie JN, Richardson BA, et al. Antenatal couple counseling increases uptake of interventions to prevent HIV-1 transmission. J Acquir Immune Defic Syndr. 2004; 37(5):1620 1626. [PubMed: 15577420]

11. Aluisio AR, Bosire R, Bourke B, et al. Male Partner Participation in Antenatal Clinic Services is Associated With Improved HIV-Free Survival Among Infants in Nairobi, Kenya: A Prospective Cohort Study. J Acquir Immune Defic Syndr. 2016; 73(2):169-176. [PubMed: 27124363]

12. Newman L, Kamb M, Hawkes S, et al. Global estimates of syphilis in pregnancy and associated adverse outcomes: analysis of multinational antenatal surveillance data. PLoS Med. 2013; 10(2):e1001396. [PubMed: 23468598]

13. Wijesooriya NS, Rochat RW, Kamb ML, et al. Global burden of maternal and congenital syphilis in 2008 and 2012: a health systems modelling study. Lancet Glob Health. 2016; 4(8):e525-533. [PubMed: 27443780]

14. Tucker JD, Bien CH, Peeling RW. Point-of-care testing for sexually transmitted infections: recent advances and implications for disease control. Curr Opin Infect Dis. 2013; 26(1):73-79. [PubMed: 23242343] 
15. Kamb ML, Newman LM, Riley PL, et al. A road map for the global elimination of congenital syphilis. Obstet Gynecol Int. 2010; 2010

16. Gichangi P, Fonck K, Sekande-Kigondu C, et al. Partner notification of pregnant women infected with syphilis in Nairobi, Kenya. Int J STD AIDS. 2000; 11(4):257-261. [PubMed: 10772091]

17. Strasser S, Bitarakwate E, Gill M, et al. Introduction of rapid syphilis testing within prevention of mother-to-child transmission of HIV programs in Uganda and Zambia: a field acceptability and feasibility study. J Acquir Immune Defic Syndr. 2012; 61(3):e40-46. [PubMed: 22820810]

18. Terris-Prestholt F, Watson-Jones D, Mugeye K, et al. Is antenatal syphilis screening still cost effective in sub-Saharan Africa. Sex Transm Infect. 2003; 79(5):375-381. [PubMed: 14573832]

19. Bristow CC, Larson E, Anderson LJ, Klausner JD. Cost-effectiveness of HIV and syphilis antenatal screening: a modelling study. Sex Transm Infect. 2016

20. Kahn JG, Jiwani A, Gomez GB, et al. The cost and cost-effectiveness of scaling up screening and treatment of syphilis in pregnancy: a model. PLoS One. 2014; 9(1):e87510. [PubMed: 24489931]

21. Brusamento S, Ghanotakis E, Tudor Car L, van-Velthoven MH, Majeed A, Car J. Male involvement for increasing the effectiveness of prevention of mother-to-child HIV transmission (PMTCT) programmes. Cochrane Database Syst Rev. 2012; 10:CD009468. [PubMed: 23076959]

22. Sabapathy K, Van den Bergh R, Fidler S, Hayes R, Ford N. Uptake of home-based voluntary HIV testing in sub-Saharan Africa: a systematic review and meta-analysis. PLoS Med. 2012; 9(12):e1001351. [PubMed: 23226107]

23. Osoti AO, John-Stewart G, Kiarie JN, et al. Home-based HIV testing for men preferred over clinicbased testing by pregnant women and their male partners, a nested cross-sectional study. BMC Infect Dis. 2015; 15:298. [PubMed: 26223540]

24. Rajput ZA, Mbugua S, Amadi D, et al. Evaluation of an Android-based mHealth system for population surveillance in developing countries. J Am Med Inform Assoc. 2012; 19(4):655-659. [PubMed: 22366295]

25. Ribeiro LV, Sabido M, Galban E, et al. Home-based counseling and testing for HIV and syphilis an evaluation of acceptability and quality control, in remote Amazonas State, Brazil. Sex Transm Infect. 2015; 91(2):94-96. [PubMed: 25305212]

26. Otieno-Nyunya B, Bennett E, Bunnell R, et al. Epidemiology of syphilis in Kenya: results from a nationally representative serological survey. Sex Transm Infect. 2011; 87(6):521-525. [PubMed: 21917697]

27. Peeling RW, Mabey D, Fitzgerald DW, Watson-Jones D. Avoiding HIV and dying of syphilis. Lancet. 2004; 364(9445):1561-1563. [PubMed: 15519615]

28. Gomez GB, Kamb ML, Newman LM, Mark J, Broutet N, Hawkes SJ. Untreated maternal syphilis and adverse outcomes of pregnancy: a systematic review and meta-analysis. Bull World Health Organ. 2013; 91(3):217-226. [PubMed: 23476094]

29. Swartzendruber A, Steiner RJ, Adler MR, Kamb ML, Newman LM. Introduction of rapid syphilis testing in antenatal care: A systematic review of the impact on HIV and syphilis testing uptake and coverage. Int J Gynaecol Obstet. 2015; 130(Suppl 1):S15-21. [PubMed: 26001704] 


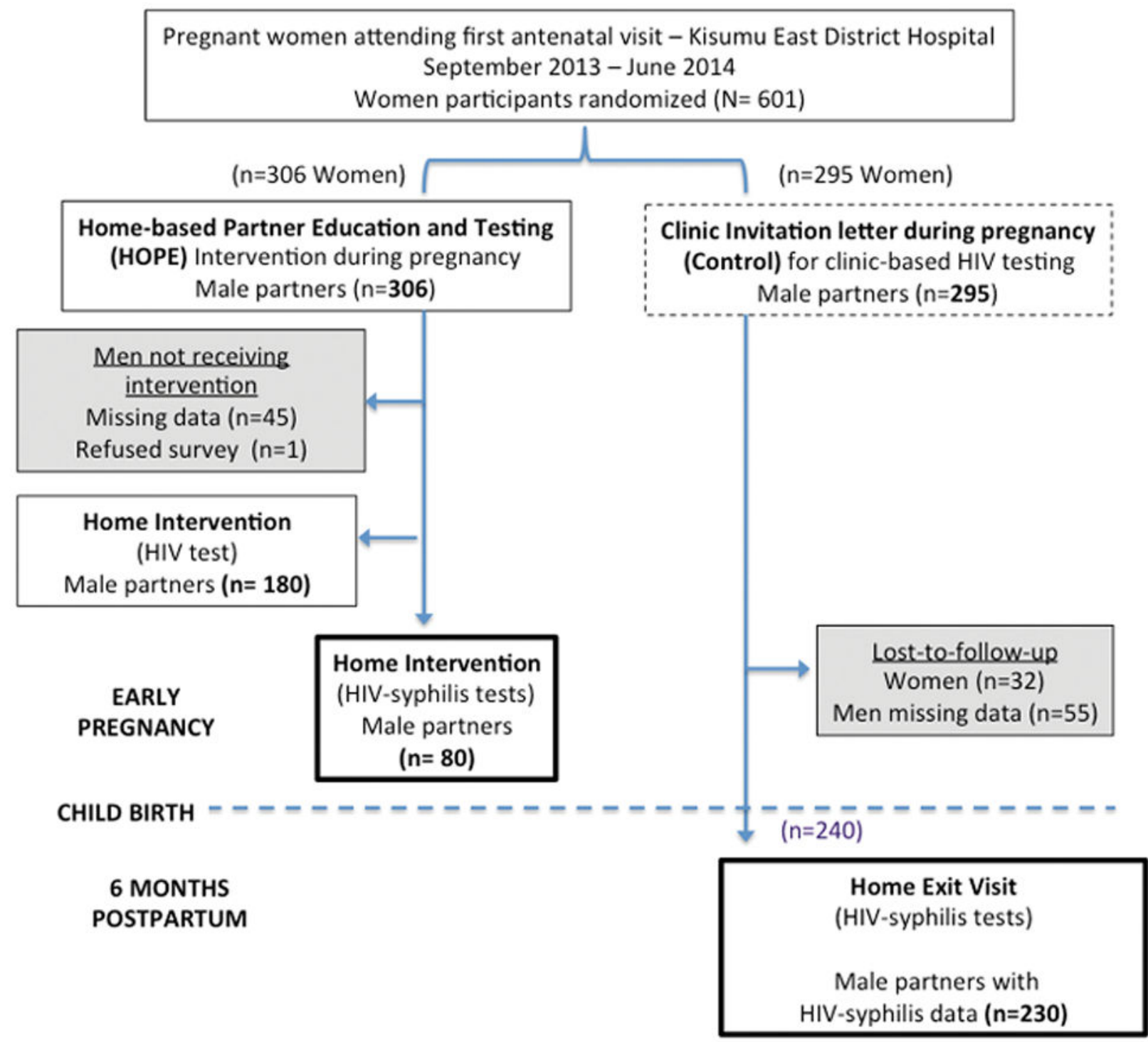

Figure 1. 


\section{Table 1}

Characteristics of male partners offered first-time home-based couple education and combined HIV-syphilis testing during pregnancy or postpartum

\begin{tabular}{|c|c|c|c|c|}
\hline \multirow[t]{2}{*}{ Baseline characteristics * } & \multicolumn{2}{|c|}{ Pregnancy Intervention Arm (N=80) } & \multicolumn{2}{|c|}{ Postpartum Control Arm (N=163) $\lambda$} \\
\hline & Median & $(\mathbf{I Q R})$ & Median & (IQR) \\
\hline Age (years) & 30 & $(27,34)$ & 30 & $(27,37)$ \\
\hline Age of sexual debut (years) ${ }^{\dagger}$ & 18 & $(16,20)$ & 17 & $(16,19)$ \\
\hline Age at marriage, if married ${ }^{\dagger}$ & 25 & $(22,28)$ & 25 & $(23,30)$ \\
\hline Years married with participant & 4 & $(1,7)$ & 4.5 & $(3,7)$ \\
\hline Number of total children ${ }^{\dagger}$ & 2 & $(1,2)$ & 2 & $(2,3)$ \\
\hline \multirow[t]{2}{*}{ Children with female participant ${ }^{\dagger}$} & 1 & $(0,2)$ & 1 & $(0,2)$ \\
\hline & $\mathbf{n}$ & $(\%)$ & $\mathbf{n}$ & $(\%)$ \\
\hline \multicolumn{5}{|l|}{ Marital status } \\
\hline Married, living together & 76 & (95) & 147 & (90) \\
\hline Married, not living together & 4 & $(5)$ & 16 & (10) \\
\hline \multicolumn{5}{|l|}{ Parenting experience ${ }^{\dagger}$} \\
\hline First-time parent & 20 & (25) & 36 & (22) \\
\hline Any previous children & 60 & (75) & 127 & (78) \\
\hline Attended ANC last pregnancy $(\mathrm{n}=52, \mathrm{n}=127)^{\dagger}$ & 11 & $(21)$ & 20 & (16) \\
\hline \multicolumn{5}{|l|}{ Education } \\
\hline No formal education & 2 & $(<1)$ & 0 & (0) \\
\hline Some primary school & 107 & $(40)$ & 40 & (25) \\
\hline Some secondary school & 108 & (40) & 94 & (58) \\
\hline$>$ Secondary school & 32 & (18) & 29 & (18) \\
\hline Previous HIV testing experience & 74 & (92) & 143 & (88) \\
\hline Reports being HIV positive ${ }^{\dagger}$ & 6 & $(8)$ & 18 & (11) \\
\hline Knows female partner's HIV status ${ }^{\dagger}$ & 65 & (81) & 149 & (93) \\
\hline Female partner is $\mathrm{HIV}+(\mathrm{n}=65, \mathrm{n}=149)$ & 11 & (17) & 20 & (13) \\
\hline
\end{tabular}

Eligibility rate total: $100 \%$, Participation rate total: $83 \%$

$\mathrm{IQR}=$ interquartile range, $\mathrm{ANC}=$ antenatal care

$\lambda_{67 \text { of } 230 \text { missing in Control arm }}$

* No significant differences between all arms $(\mathrm{p}<0.05)$

${ }^{\dagger} 5$ men in Control missing age of sexual debut; 1 in Control missing age at marriage; 2 in Control missing number total children; 3 in Control missing number children with female participant; 8 in Intervention missing attended antenatal care during the previous pregnancy; 1 in Intervention missing HIV status; 3 in Control missing female partner's HIV status. 


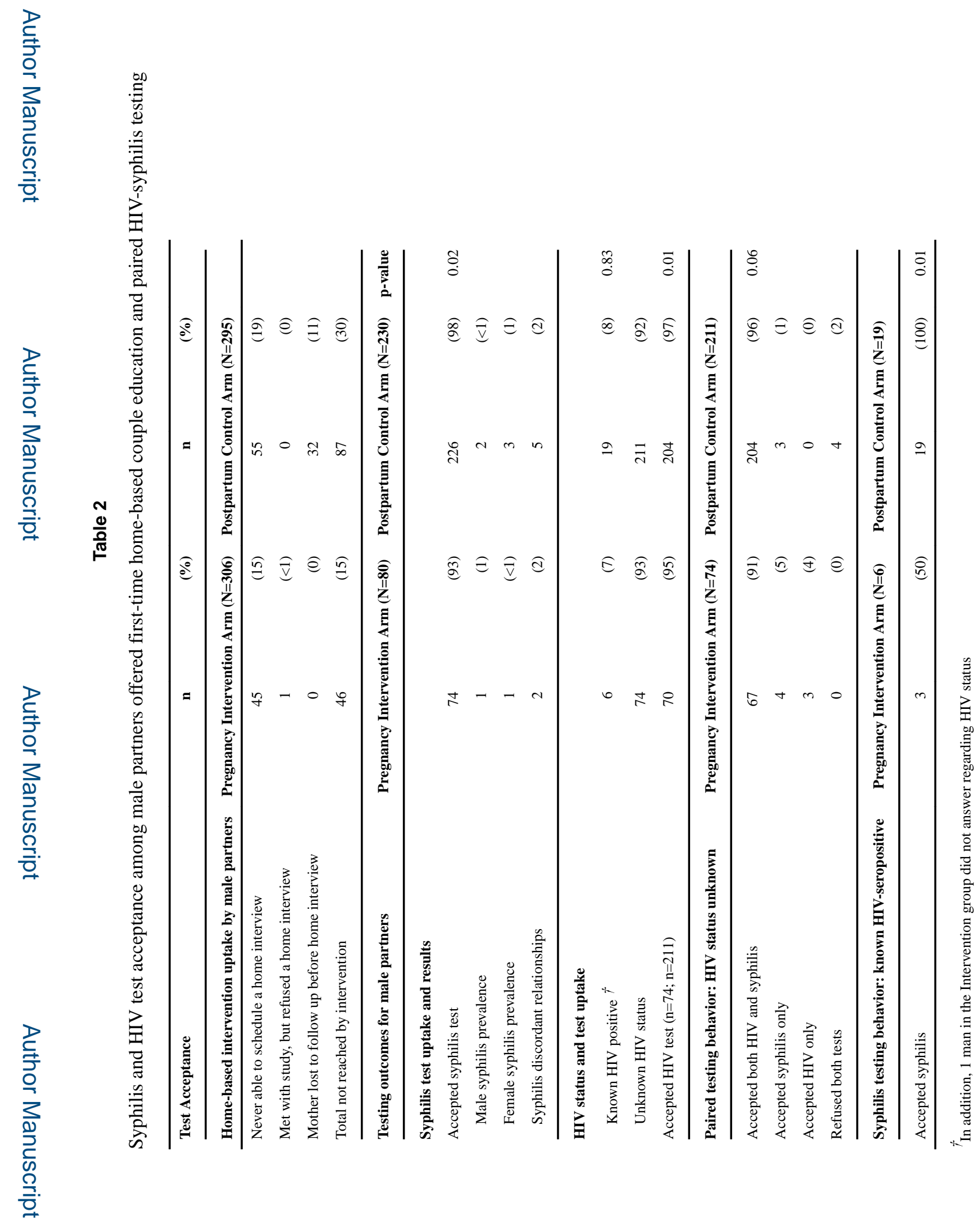

Sex Transm Dis. Author manuscript; available in PMC 2018 September 01. 


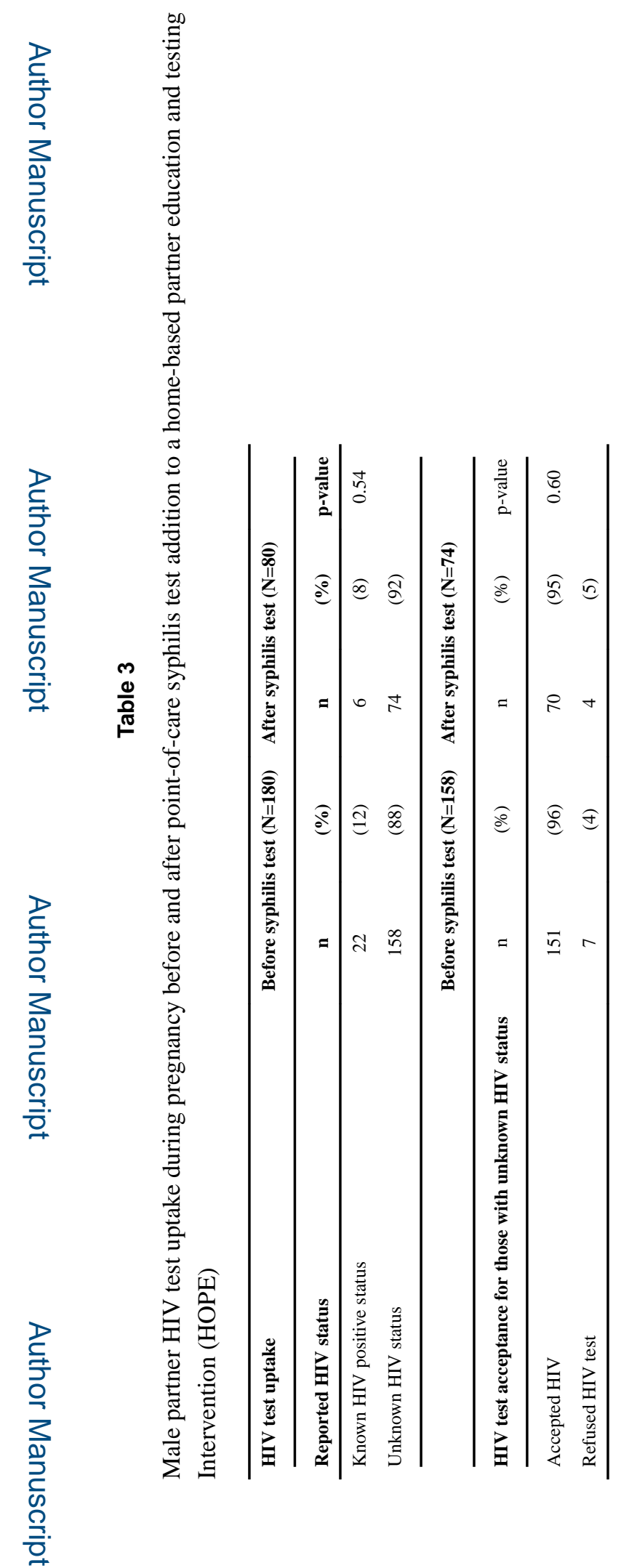

Sex Transm Dis. Author manuscript; available in PMC 2018 September 01. 